\title{
Fekete-Szegö Inequalities of a Subclass of Multivalent Analytic Functions
}

C. Selvaraj, K. R. Karthikeyan, and S. Lakshmi

\begin{abstract}
The main object of this paper is to study Fekete-Szegö problem for a certain subclass of $p$ - valent analytic functions. Fekete-Szegö inequality of several classes are obtained as special cases from our results. Applications of the result are also obtained on the class defined by convolution.
\end{abstract}

AMS Subject Classification (2000). 30C45.

Keywords. Fekete-Szegö inequlities, $p$-valent functions, Convolution, Fractional derivatives

\section{INTRODUCTION}

Let $\mathcal{A}$ denote the class of all analytic function of the form

$$
f(z)=z+\sum_{n=2}^{\infty} a_{n} z^{n},
$$

in the open unit disc $\mathcal{U}=\{z: z \in \mathbb{C} ;|z|<1\}$. Let $\mathcal{S}$ be the subclass of $\mathcal{A}$ consisting of univalent functions in $\mathcal{U}$. We denote by $\mathcal{S}^{*}, \mathcal{C}$ and $\mathcal{K}$ the familiar subclasses of $\mathcal{A}$ consisting of functions which are respectively starlike, convex and close-to-convex in $\mathcal{U}$. Our favorite references of the field are $[5,6]$ which covers most of the topics in a lucid and economical style. The well-known 
example in these classes is the Koebe function, $k(z)$ defined by

$$
k(z)=\frac{z}{(1-z)^{2}}=z+\sum_{n=2}^{\infty} n z^{n} .
$$

The Bieberbach conjecture about the coefficients of the univalent functions of the unit disc was formulated by Bieberbach [1] in the year 1916. The conjecture states that for every function $f(z) \in \mathcal{S}$, given by (1.1), we have $\left|a_{n}\right| \leq n$, for every $n$. Strict inequality holds for all $n$ unless $f$ is the Koebe function or one of its rotation. For many years, this conjecture remained as a challenge to mathematicians. After the proof of $\left|a_{3}\right| \leq 3$ by Löwner in 1923, Fekete and Szegö [4] surprised mathematicians by showing that the complicated inequality

$$
\left|a_{3}-\mu a_{2}^{2}\right| \leq 1+2 \exp \left(\frac{-2 \mu}{1-\mu}\right)
$$

holds and is best possible for each $0 \leq \mu \leq 1$. Note that this coefficient region was thoroughly investigated by Schaeffer and Spencer [23].

For a class of function in $\mathcal{A}$ and a real (or, more generally, a complex) number $\mu$, the Fekete-Szegö problem is all about finding the best possible constant $C(\mu)$ so that $\left|a_{3}-\mu a_{2}^{2}\right| \leq C(\mu)$ for every function in $\mathcal{A}$. Many papers have been devoted to this problem see $[2,3,10,12,13,26]$. In this paper, we shall obtain Fekete-Szegö bounds for the functions in the class $\mathcal{C}_{p, q}^{n, \gamma}(\phi)$.

For $f$ and $g$ analytic in $\mathcal{U}$, we say that the function $f$ is subordinate to $g$ if there exists a Schwarz function $w$, analytic in $\mathcal{U}$ with $w(0)=0$ and $|w(z)|<1$ such that $f(z)=g(w(z))$ for $z \in \mathcal{U}$. We denote it by $f(z) \prec g(z)$. If $g$ is univalent in $\mathcal{U}$, then the subordination is equivalent to $f(0)=g(0)$ and $f(\mathcal{U}) \subset g(\mathcal{U})$. For the functions $f(z)$ and $g(z)$ belonging to $\mathcal{A}$, we say that $f(z)$ is majorized by $g(z)$ in $\mathcal{U}$ and write $f(z) \ll g(z),(z \in \mathcal{U})$, if there exists a function $\phi(z)$, analytic in $\mathcal{U}$, such that

$$
|\phi(z)| \leq 1 \text { and } f(z)=\phi(z) g(z), \quad(z \in \mathcal{U}) .
$$

One of the very interesting generalization of the function class $\mathcal{S}^{*}$ is the so called starlike functions of complex order $\gamma$ which satisfies the condition

$$
1+\frac{1}{\gamma}\left(\frac{z f^{\prime}(z)}{f(z)}-1\right) \prec \phi(z), \quad(f \in \mathcal{A}),
$$

where $\phi \in \mathcal{P}$, the class of functions with positive real part and we denote it by $\mathcal{S}_{\gamma}(\phi)$. Similarly, let $\mathcal{C}_{\gamma}(\phi)$ denote the class of functions in $\mathcal{A}$ satisfying 
the condition

$$
1+\frac{1}{\gamma} \frac{z f^{\prime \prime}(z)}{f(z)} \prec \phi(z), \quad(f \in \mathcal{A}) .
$$

Note that $\mathcal{S}_{\gamma}(1+z / 1-z)=\mathcal{S}_{\gamma}$ and $\mathcal{C}_{\gamma}(1+z / 1-z)=\mathcal{C}_{\gamma}$ are the classes considered by Nasr and Aouf in [14] and by Wiatrowski in [27].

The convolution or Hadamard product of two analytic functions $f, g \in \mathcal{A}$ where $f(z)=z+\sum_{n=2}^{\infty} a_{n} z^{n}$ and $g(z)=z+\sum_{n=2}^{\infty} b_{n} z^{n}$ is given by

$$
(f * g)(z)=z+\sum_{n=2}^{\infty} a_{n} b_{n} z^{n} .
$$

Let $\mathcal{A}_{p}$ be the class of functions $f(z)$ of the form

$$
f(z)=z^{p}+\sum_{j=p+1}^{\infty} a_{j} z^{j} \quad(p \in N=\{1,2,3, \ldots\}),
$$

which are analytic and $p$-valent in the unit disk $\mathcal{U}$. Sălăgean [21] has introduced the following operator, which is popularly called the Sălăgean operator.

$$
\begin{aligned}
D^{0} f(z) & =f(z), \\
D^{1} f(z) & =z f^{\prime}(z) \\
\vdots & \vdots \quad \vdots \\
D^{n} f(z) & =D\left(D^{n-1} f(z)\right), \quad(n \in N) .
\end{aligned}
$$

Note that if $f(z) \in \mathcal{A}_{p}$, then

$$
D^{n} f(z)=p^{n} z^{p}+\sum_{j=p+1}^{\infty} j^{n} a_{j} z^{j}
$$

Let $f^{(q)}(z)$ denote the $q$-order ordinary differential operator, for a function $f(z) \in \mathcal{A}(p)$,

$$
\begin{gathered}
f^{(q)}(z)=\frac{p !}{(p-q) !} z^{p-q}+\sum_{j=p+1}^{\infty} \frac{j !}{(j-q) !} a_{j} z^{j-q}, \\
\left(p>q, \quad p \in N, \quad q \in N_{0}=N \cup\{0\}\right) .
\end{gathered}
$$

It can be easily seen that

$$
D^{n} f^{(q)}(z)=(p-q)^{n} \frac{p !}{(p-q) !} z^{p-q}+\sum_{j=p+1}^{\infty}(j-q)^{n} \frac{j !}{(j-q) !} a_{j} z^{j-q} .
$$

Motivated by [20], we define the following. 
Definition 1.1. Let $\phi(z)$ be analytic in $\mathcal{U}$ with $\phi(0)=1$. A function $f(z) \in$ $\mathcal{A}_{p}$ is said to be in the class $\mathcal{C}_{p, q}^{n, \gamma}(\phi)$ of p-valently anlytic functions of complex order $\gamma \neq 0$ in $\mathcal{U}$ if and only if

$$
\begin{gathered}
1+\frac{1}{\gamma}\left[\frac{D^{n+1} f^{(q)}(z)}{D^{n} f^{(q)}(z)}-p+q\right] \prec \phi(z), \\
\left(z \in \mathcal{U}, \quad p \in N, \quad n, q \in N_{0}=N \cup\{0\}, \quad \gamma \in \mathbb{C}-\{0\}\right) .
\end{gathered}
$$

Remark 1.1. We note that by specializing the parameters $n, p, q$ and $\gamma$, we obtain several classes of analytic functions. Here we list few of them.

1. If $p=1, q=1$, then the class $\mathcal{C}_{p, q}^{n, \gamma}(\phi)$ reduces to the class studied by Attiya [8].

2. If we let $\phi(z)=\frac{1+z}{1-M z}$, then the class $\mathcal{C}_{p, q}^{n, \gamma}(\phi)$ reduces to the class introduced and studied by Aouf, Darwish and Attiya in [7].

Apart from the above, several other well known and new classes of analytic functions can be obtained by specializing the parameters involved.

\section{FEKETE-SZEGÖ INEQUALITY}

To prove our result, we need the following Lemmas.

Lemma 2.1. [12] Let $p(z)=1+c_{1} z+c_{2} z^{2}+\cdots$ is analytic function with positive real part in $\mathcal{U}$ and $v$ is complex number, then

$$
\left|c_{2}-v c_{1}^{2}\right| \leq 2 \max \{1,|2 v-1|\},
$$

the result is sharp for functions given by

$$
p(z)=\frac{1+z^{2}}{1-z^{2}}, \quad p(z)=\frac{1+z}{1-z} .
$$

Lemma 2.2. [12] Let $p(z)=1+c_{1} z+c_{2} z^{2}+\cdots$ is analytic function with positive real part in $\mathcal{U}$, then

$$
\left|c_{2}-v c_{1}^{2}\right| \leq\left\{\begin{array}{lll}
-4 v+2, & \text { if } & v \leq 0 \\
2, & \text { if } & 0 \leq v \leq 1 \\
4 v-2, & \text { if } & v \geq 1
\end{array}\right.
$$


When $v<0$ or $v>1$ the equality holds if and only if $p(z)=(1+z) /(1-z)$ or one of its rotations. If $0<v<1$, then the equality if and only if $p(z)=$ $\left(1+z^{2}\right) /\left(1-z^{2}\right)$ or one of its rotations. If $v=0$ the equality holds if and only if

$$
p(z)=\left(\frac{1}{2}+\frac{1}{2} \vartheta\right) \frac{1+z}{1-z}+\left(\frac{1}{2}-\frac{1}{2} \vartheta\right) \frac{1-z}{1+z},(0 \leq \vartheta \leq 1),
$$

or one of its rotations. If $v=1$ the equality holds if and only if

$$
\frac{1}{p(z)}=\left(\frac{1}{2}+\frac{1}{2} \vartheta\right) \frac{1+z}{1-z}+\left(\frac{1}{2}-\frac{1}{2} \vartheta\right) \frac{1-z}{1+z},(0 \leq \vartheta \leq 1) .
$$

Also the above upper bound is sharp and it can be improved as follows when $0 \leq v \leq 1$

$$
\begin{gathered}
\left|c_{2}-v c_{1}^{2}\right|+v\left|c_{1}\right|^{2} \leq 2, \quad(0<v \leq 1 / 2), \\
\left|c_{2}-v c_{1}^{2}\right|+(1-v)\left|c_{1}\right|^{2} \leq 2, \quad(1 / 2 \leq v<1) .
\end{gathered}
$$

Theorem 2.3. Let $\phi(z)=1+B_{1} z+B_{2} z^{2}+\cdots\left(B_{1} \neq 0\right)$. If $f(z) \in \mathcal{C}_{p, q}^{n, \gamma}(\phi)$, then

$$
\left|a_{p+2}-\mu a_{p+1}^{2}\right| \leq|\gamma| \frac{(p-q+1)(p-q)^{n}}{(p-q+2)^{n-1}(p+1)(p+2)} \frac{\left|B_{1}\right|}{2} \max \left\{1 ;\left|\frac{B_{2}}{B_{1}}+2 k_{1}\right|\right\}
$$

where

$k_{1}=\left(\frac{(p-q+1)^{2 n-1}(p-q)^{n}(p+1)}{2(p-q+2)^{n-1}(p+2)}-\mu\right) \frac{B_{1} \gamma(p-q)^{n}(p-q+2)^{n-1}(p+2)}{(p-q+1)^{2 n-1}(p+1)}$.

Proof. Let $f(z) \in \mathcal{C}_{p, q}^{n, \gamma}(\phi)$, then there exists a Schwarz function $\omega(z)$ in $\mathcal{U}$ with $\omega(0)=0$ and $|\omega(z)|<1$ in $\mathcal{U}$, such that

$$
\frac{D^{n+1} f^{(q)}(z)}{D^{n} f^{(q)}(z)}-p+q=\phi(\omega(z)) .
$$

Define the function $p(z)$ by

$$
p(z)=\frac{1+\omega(z)}{1-\omega(z)}=1+c_{1} z+c_{2} z^{2}+\cdots, z \in \mathcal{U} .
$$

Since $\omega(z)$ is a Schwarz function, it can be easily seen that $\operatorname{Re}\left(\frac{1+\omega(z)}{1-\omega(z)}\right)>0$. That is $\operatorname{Re}(p(z))>0$ and $p(0)=1$. 
Therefore

$$
\begin{aligned}
\phi(\omega(z)) & =\phi\left(\frac{p(z)-1}{p(z)+1}\right) \\
& =\phi\left(\frac{1}{2}\left[c_{1} z+\left(c_{2}-\frac{c_{1}^{2}}{2}\right) z^{2}+\left(c_{3}-c_{1} c_{2}+\frac{c_{1}^{3}}{4}\right) z^{3}+\cdots\right]\right) \\
& =1+\frac{1}{2} B_{1} c_{1} z+\left[\frac{1}{2} B_{1}\left(c_{2}-\frac{c_{1}^{2}}{2}\right)+\frac{1}{4} B_{2} c_{1}^{2}\right] z^{2}+\cdots
\end{aligned}
$$

Now by substituting (2.3) in (2.2), we have

$1+\frac{1}{\gamma}\left[\frac{D^{n+1} f^{(q)}(z)}{D^{n} f^{(q)}(z)}-p+q\right]=1+\frac{1}{2} B_{1} c_{1} z+\left[\frac{1}{2} B_{1}\left(c_{2}-\frac{c_{1}^{2}}{2}\right)+\frac{1}{4} B_{2} c_{1}^{2}\right] z^{2}+\cdots$.

From this equation, we obtain

$$
\frac{(p-q+1)^{n-1}(p+1) a_{p+1}}{\gamma(p-q)^{n}}=\frac{B_{1} c_{1}}{2}
$$

and

$$
\begin{array}{r}
\frac{2(p-q+2)^{n-1}(p+1)(p+2) a_{p+2}}{\gamma(p-q+1)(p-q)^{n}}-\frac{(p-q+1)^{2(n-1)}(p+1)^{2} a_{p+1}^{2}}{\gamma(p-q)^{2 n}}= \\
\frac{B_{1} c_{2}}{2}-\frac{B_{1} c_{1}^{2}}{4}+\frac{B_{2} c_{1}^{2}}{4} .
\end{array}
$$

Or equivalently

$$
a_{p+1}=\frac{\gamma B_{1} c_{1}(p-q)^{n}}{2(p-q+1)^{n-1}(p+1)}
$$

and

$$
\begin{aligned}
& a_{p+2}=\frac{\gamma(p-q+1)(p-q)^{n}}{2(p-q+2)^{n-1}(p+1)(p+2)} \times \\
& \left(\frac{B_{1} c_{2}}{2}-\frac{B_{1} c_{1}^{2}}{4}+\frac{B_{2} c_{1}^{2}}{4}+\frac{(p-q+1)^{2(n-1)}(p+1)^{2} a_{p+1}^{2}}{\gamma}\right) .
\end{aligned}
$$

Therefore

$$
\begin{aligned}
& a_{p+2}-\mu a_{p+1}^{2}=\frac{\gamma(p-q+1)(p-q)^{n}}{2(p-q+2)^{n-1}(p+1)(p+2)}\left(\frac{B_{1} c_{2}}{2}-\frac{B_{1} c_{1}^{2}}{4}+\frac{B_{2} c_{1}^{2}}{4}\right) \\
& +\left(\frac{(p-q+1)^{2 n-1}(p-q)^{n}(p+1)}{2(p-q+2)^{n-1}(p+2)}-\mu\right) \times\left(\frac{\gamma B_{1}^{2} c_{1}^{2}(p-q)^{2 n}}{4(p-q+1)^{2(n-1)}(p+1)^{2}}\right) .
\end{aligned}
$$


Or equivalently,

$$
a_{p+2}-\mu a_{p+1}^{2}=\frac{\gamma B_{1}(p-q+1)(p-q)^{n}}{4(p-q+2)^{n-1}(p+1)(p+2)}\left(c_{2}-\vartheta c_{1}^{2}\right)
$$

where

$$
\vartheta=\frac{1}{2}-\frac{B_{2}}{2 B_{1}}-k_{1}
$$

and

$$
k_{1}=\left(\frac{(p-q+1)^{2 n-1}(p-q)^{n}(p+1)}{2(p-q+2)^{n-1}(p+2)}-\mu\right) \frac{B_{1} \gamma(p-q)^{n}(p-q+2)^{n-1}(p+2)}{(p-q+1)^{2 n-1}(p+1)} .
$$

Our result now follows on application of Lemma2.1.

The result is sharp for the function

$$
\frac{D^{n+1} f^{(q)}(z)}{D^{n} f^{(q)}(z)}-p+q=\phi\left(z^{2}\right)
$$

and

$$
\frac{D^{n+1} f^{(q)}(z)}{D^{n} f^{(q)}(z)}-p+q=\phi(z) .
$$

This completes the proof of Theorem2.3.

Corollary 2.4. Let $f(z) \in \mathcal{A}$ satisfy the inequality

$$
\alpha<\operatorname{Re}\left\{1+\frac{1}{\gamma}\left[\frac{z f^{\prime}(z)}{f(z)}-1\right]\right\}<\beta
$$

then

$$
\left|a_{3}-\mu a_{2}^{2}\right| \leq \frac{|\gamma|(\beta-\alpha)}{\sqrt{2} \pi} \sqrt{1-\cos \left(\frac{2 \pi(1-\alpha)}{\beta-\alpha}\right)} \max \left\{1,\left|\frac{B_{2}}{B_{1}}+(1-\mu) \gamma B_{1}\right|\right\}
$$

where

$$
B_{n}=\frac{\beta-\alpha}{n \pi} i\left[1-e^{2 n \pi i((1-\alpha) /(\beta-\alpha))}\right]
$$

Proof. Let

$$
\phi(z)=1+\frac{\beta-\alpha}{\pi} i \log \left(\frac{1-e^{2 \pi i((1-\alpha) /(\beta-\alpha))} z}{1-z}\right) .
$$

Clearly, it can be seen that $\phi(z)$ maps $\mathcal{U}$ onto a convex domain conformally and is of the form

$$
h(z)=1+\sum_{n=1}^{\infty} B_{n} z^{n}
$$


where $B_{n}=\frac{\beta-\alpha}{n \pi} i\left(1-e^{2 n \pi i((1-\alpha) /(\beta-\alpha))}\right)$. From the equivalent subordination condition proved by Kuroki and Owa in [11], the inequality (2.6) can be rewritten in the form

$$
1+\frac{1}{\gamma}\left[\frac{z f^{\prime}(z)}{f(z)}-1\right] \prec \phi(z)
$$

Following the steps as in Theorem 2.3, we get the desired result.

Using the arguments similar to those detailed in the Corollary2.4, we have the following result.

Corollary 2.5. Let $f(z) \in \mathcal{A}$ satisfy the inequality

$$
\alpha<\operatorname{Re}\left\{1+\frac{1}{\gamma}\left[\frac{z f^{\prime \prime}(z)}{f(z)}\right]\right\}<\beta
$$

then

$$
\begin{aligned}
& \left|a_{3}-\mu a_{2}^{2}\right| \leq \\
& \quad \frac{|\gamma|(\beta-\alpha)}{3 \sqrt{2} \pi} \sqrt{1-\cos \left(\frac{2 \pi(1-\alpha)}{\beta-\alpha}\right)} \max \left\{1,\left|\frac{B_{2}}{B_{1}}+\left(1-\frac{3}{2} \mu\right) \gamma B_{1}\right|\right\},
\end{aligned}
$$

where

$$
B_{n}=\frac{\beta-\alpha}{n \pi} i\left(1-e^{2 n \pi i((1-\alpha) /(\beta-\alpha))}\right) .
$$

If we let $n=0, p=1$ and $q=0$ in Theorem2.3, we get the desired result.

Corollary 2.6. [19] Let $\phi(z)=1+B_{1} z+B_{2} z^{2}+\cdots$ with $\left.B_{1} \neq 0\right)$. If $f(z) \in \mathcal{S}_{\gamma}(\phi)$, then

$$
\left|a_{3}-\mu a_{2}^{2}\right| \leq \frac{|\gamma|\left|B_{1}\right|}{2} \max \left\{1,\left|\frac{B_{2}}{B_{1}}+(1-2 \mu) \gamma B_{1}\right|\right\} .
$$

The result is sharp.

If we let $n=1, p=1$ and $q=0$ in Theorem2.3, we get the following result.

Corollary 2.7. Let $\phi(z)=1+B_{1} z+B_{2} z^{2}+\cdots$ with $\left.B_{1} \neq 0\right)$. If $f(z) \in$ $\mathrm{e}_{\gamma}(\phi)$, then

$$
\left|a_{3}-\mu a_{2}^{2}\right| \leq \frac{|\gamma|\left|B_{1}\right|}{6} \max \left\{1,\left|\frac{B_{2}}{B_{1}}+\left(1-\frac{3}{2} \mu\right) \gamma B_{1}\right|\right\} .
$$

The result is sharp. 
Theorem 2.8. Let $\phi(z)=1+B_{1} z+B_{2} z^{2}+\cdots\left(B_{1}>0\right)$. If $f(z)$ given by (1.3) belongs to $\mathcal{C}_{p, q}^{n, \gamma}(\phi)$, then

$$
\left|a_{p+2}-\mu a_{p+1}^{2}\right| \leq\left\{\begin{array}{lll}
2 \gamma k_{1}\left[B_{1}^{2}+B_{2}-\mu \gamma B_{1}^{2} k_{2}\right], & \text { if } & \mu \leq \sigma_{1} \\
2 \gamma B_{1} k_{1}, & \text { if } & \sigma_{1} \leq \mu \leq \sigma_{2} \\
-2 \gamma k_{1}\left[B_{1}^{2}+B_{2}-\mu B_{1}^{2} \gamma k_{2}\right], & \text { if } & \mu \geq \sigma_{2},
\end{array}\right.
$$

where

$$
\begin{gathered}
\sigma_{1}=\frac{1}{\gamma B_{1} k_{2}}\left[-1+\frac{B_{2}}{B_{1}}+B_{1}\right] \\
\sigma_{2}=\frac{1}{\gamma B_{1} k_{2}}\left[1+\frac{B_{2}}{B_{1}}+B_{1}\right] \\
k_{1}=\frac{(p-q)^{n}(p-q+2) !}{4(p-q+2)^{n}(p+2)(p+1)(p-q) !}
\end{gathered}
$$

and

$$
k_{2}=\frac{2(p-q+2)^{n}(p-q)^{n}(p+2)(p-q) !}{(p-q+1)^{n-2}(p-q+2) !} .
$$

Proof. Applying Lemma 2.2 to (2.4) and (2.5), we can obtain our results. To show that these bounds are sharp, we define the functions $K_{\phi_{n}},(n \geq 2)$ by

$$
\begin{gathered}
\operatorname{Re}\left\{1+\frac{1}{\gamma}\left[\frac{D^{n+1} K_{\phi_{n}}^{(q)}(z)}{D^{n} K_{\phi_{n}}^{(q)}(z)}-p+q\right]\right\}=\phi\left(z^{n-1}\right) . \\
K_{\phi_{n}}(0)=0=K_{\phi_{n}}^{\prime}(0)-1,
\end{gathered}
$$

and the functions $F_{\beta}$ and $G_{\beta}(0 \leq \beta \leq 1)$ by

$$
\begin{gathered}
\operatorname{Re}\left\{1+\frac{1}{\gamma}\left[\frac{D^{n+1} F_{\beta}^{(q)}(z)}{D^{n} F_{\beta}^{(q)}(z)}-p+q\right]\right\}=\phi\left(\frac{z(z+\beta)}{1+\beta z}\right), \\
F_{\beta}(0)=0=F_{\beta}^{\prime}(0)-1,
\end{gathered}
$$

and

$$
\begin{gathered}
\operatorname{Re}\left\{1+\frac{1}{\gamma}\left[\frac{D^{n+1} G_{\beta}^{(q)}(z)}{D^{n} G_{\beta}^{(q)}(z)}-p+q\right]\right\}=\phi\left(\frac{-z(z+\beta)}{1+\beta z}\right), \\
G_{\beta}(0)=0=G_{\beta}^{\prime}(0)-1 .
\end{gathered}
$$

Clearly, the functions $K_{\phi_{n}}, F_{\beta}$ and $G_{\beta} \in \mathcal{C}_{p, q}^{n, \gamma}(\phi)$. Also we write $K_{\phi}:=K_{\phi_{2}}$, if $\mu<\sigma_{1}$ or $\mu>\sigma_{2}$, then the equality holds if and only if $f$ is $K_{\phi}$ or one of 
its rotations. When $\sigma_{1}<\mu<\sigma_{2}$ then the equality holds if and only if $f$ is $K_{\phi_{3}}$ or one of its rotations. $\mu=\sigma_{1}$ then the equality holds if and only if $f$ is $F_{\beta}$ or one of its rotations. $\mu=\sigma_{2}$ then the equality holds if and only if $f$ is $G_{\beta}$ or one of its rotations. This completes the proof of Theorem 2.

If $\sigma_{1} \leq \mu \leq \sigma_{2}$, then in view of Lemma 2.2, Theorem 2.8 can be improved.

Theorem 2.9. Let $\phi(z)=1+B_{1} z+B_{2} z^{2}+\cdots\left(B_{1}>0\right)$. If $f(z)$ given by(1.3) belongs to $\mathcal{C}_{p, q}^{n, \gamma}(\phi)$ and $\sigma_{3}$ is given by

$$
\sigma_{3}=\frac{1}{\gamma B_{1} k_{2}}\left[B_{1}+\frac{B_{2}}{B_{1}}\right]
$$

If $\sigma_{1}<\mu \leq \sigma_{3}$, then

$$
\left|a_{p+2}-\mu a_{p+1}^{2}\right|+\frac{1}{\gamma B_{1} k_{2}}\left[\left(1-B_{1}\right)-\frac{B_{2}}{B_{1}}+\mu \gamma B_{1} k_{2}\right]\left|a_{p+1}^{2}\right| \leq 2 \gamma B_{1} k_{1} .
$$

If $\sigma_{3}<\mu \leq \sigma_{2}$, then

$$
\left|a_{p+2}-\mu a_{p+1}^{2}\right|+\frac{1}{\gamma B_{1} k_{2}}\left[\left(1+B_{1}\right)+\frac{B_{2}}{B_{1}}-\mu \gamma B_{1} k_{2}\right]\left|a_{p+1}^{2}\right| \leq 2 \gamma B_{1} k_{1} .
$$

Taking $n=0, p=1$ and $q=0$, we obtain the following result for the functions belonging to the class $\mathrm{e}_{\gamma}(\phi)$.

Corollary 2.10. (see [24]) Let $\phi(z)=1+B_{1} z+B_{2} z^{2}+\cdots$ with $B_{1}>0$ and $B_{2} \geq 0$. Let

$$
\sigma_{4}=\frac{\gamma B_{1}^{2}+\left(B_{2}-B_{1}\right)}{2 \gamma B_{1}^{2}}, \quad \sigma_{5}=\frac{\gamma B_{1}^{2}+\left(B_{2}+B_{1}\right)}{2 \gamma B_{1}^{2}}, \quad \sigma_{6}=\frac{\gamma B_{1}^{2}+B_{2}}{2 \gamma B_{1}^{2}} .
$$

If $f(z)$ given by (1.1) belongs to the class $\mathcal{S}_{\gamma}(\phi)$ with $\gamma>0$, then

$$
\left|a_{3}-\mu a_{2}^{2}\right| \leq\left\{\begin{array}{lll}
\frac{B_{2} \gamma}{2}+\frac{B_{1}^{2} \gamma^{2}}{2}(1-2 \mu) & \text { if } & \mu \leq \sigma_{4} \\
\frac{B_{1} \gamma}{2} & \text { if } & \sigma_{4} \leq \mu \leq \sigma_{5} \\
-\frac{B_{2} \gamma}{2}-\frac{B_{1}^{2} \gamma^{2}}{2}(1-2 \mu) & \text { if } & \mu \geq \sigma_{5}
\end{array}\right.
$$

Further, if $\sigma_{4} \leq \mu \leq \sigma_{6}$, then

$$
\left|a_{3}-\mu a_{2}^{2}\right|+\frac{1}{2 B_{1}^{2} \gamma}\left[B_{1}-B_{2}-B_{1}^{2} \gamma(1-2 \mu)\right]\left|a_{2}\right|^{2} \leq \frac{B_{1} \gamma}{2} .
$$


If $\sigma_{6} \leq \mu \leq \sigma_{5}$, then

$$
\left|a_{3}-\mu a_{2}^{2}\right|+\frac{1}{2 B_{1}^{2} \gamma}\left[B_{1}+B_{2}+B_{1}^{2} \gamma(1-2 \mu)\right]\left|a_{2}\right|^{2} \leq \frac{B_{1} \gamma}{2} .
$$

The result is sharp.

Taking $n=1, p=1$ and $q=0$, we obtain the following result for the functions belonging to the class $\mathcal{C}_{\gamma}(\phi)$.

Corollary 2.11. (see [24]) Let $\phi(z)=1+B_{1} z+B_{2} z^{2}+\cdots$ with $B_{1}>0$ and $B_{2} \geq 0$. Let

$$
\chi_{1}=\frac{2\left[\gamma B_{1}^{2}+B_{2}-B_{1}\right]}{\gamma B_{1}^{2}}, \quad \chi_{2}=\frac{2\left[\gamma B_{1}^{2}+B_{2}+B_{1}\right]}{\gamma B_{1}^{2}}, \quad \chi_{3}=\frac{2\left[\gamma B_{1}^{2}+B_{2}\right]}{\gamma B_{1}^{2}} .
$$

If $f$ given by (1.1) belongs to the class $\mathrm{C}_{\gamma}(\phi)$ with $\gamma>0$, then

$$
\left|a_{3}-\mu a_{2}^{2}\right| \leq\left\{\begin{array}{lll}
\frac{B_{2} \gamma}{6}+\frac{B_{1}^{2} \gamma^{2}}{6}\left(1-\frac{3}{2} \mu\right) & \text { if } & \mu \leq \chi_{1} ; \\
\frac{B_{1} \gamma}{6} & \text { if } & \chi_{1} \leq \mu \leq \chi_{2} \\
-\frac{B_{2} \gamma}{6}-\frac{B_{1}^{2} \gamma^{2}}{6}\left(1-\frac{3}{2} \mu\right) & \text { if } & \mu \geq \chi_{3} .
\end{array}\right.
$$

Further, if $\chi_{1} \leq \mu \leq \chi_{3}$, then

$$
\left|a_{3}-\mu a_{2}^{2}\right|+\frac{2}{3 B_{1}^{2} \gamma}\left[B_{1}-B_{2}-B_{1}^{2} \gamma\left(1-\frac{3}{2} \mu\right)\right]\left|a_{2}\right|^{2} \leq \frac{B_{1} \gamma}{6} .
$$

If $\chi_{2} \leq \mu \leq \chi_{3}$, then

$$
\left|a_{3}-\mu a_{2}^{2}\right|+\frac{2}{3 B_{1}^{2} \gamma}\left[B_{1}+B_{2}+B_{1}^{2} \gamma\left(1-\frac{3}{2} \mu\right)\right]\left|a_{2}\right|^{2} \leq \frac{B_{1} \gamma}{6} .
$$

The result is sharp.

\section{Application to Functions Defined by Fractional Deriva- tives}

In view of defining the extended fractional differintegral operator, we begin by recalling here the following definitions.

The hypergeometric function which is the solution of the homogeneous hypergeometric differential equation

$$
z(1-z) w^{\prime \prime}(z)+[c-(a+b+1) z] w^{\prime}(z)-a b w(z)=0
$$


is one of the most important special functions, because of its many connections to other classes of special functions, its numerous identities and expressions in terms of series and integrals.

For parameters $a, b, c \in \mathbb{C}$, with $c \neq 0,-1,-2, \ldots$, and $z \in \mathbb{C}$ with $|z|<1$, the (Gaussian) hypergeometric function is defined by

$$
F(a, b ; c ; z)={ }_{2} F_{1}(a, b ; c ; z)=\sum_{n=0}^{\infty} \frac{(a)_{n}(b)_{n}}{(c)_{n}} \frac{z^{n}}{n !} .
$$

The series on the right-hand side, called the hypergeometric series, is convergent for $|z|<1$. The $(x)_{k}$ is the Pochhammer symbol defined, in terms of the Gamma function $\Gamma$, by

$$
(x)_{k}=\frac{\Gamma(x+k)}{\Gamma(x)}= \begin{cases}1 & \text { if } k=0 \\ x(x+1)(x+2) \ldots(x+k-1) & \text { if } k \in N .\end{cases}
$$

Fractional calculus is a field of mathematic study that grows out of the traditional definitions of the calculus integral and derivative operators in much the same way fractional exponents is an outgrowth of exponents with integer value. For details, we refer to [9,22]. From among the various definitions for fractional calculus (i.e., fractional derivatives and fractional integrals) given in the literature, we have to recall here the following definitions for fractional calculus which are studied by Owa $[15,16]$ and by Owa and Srivastava [17].

Definition 3.1. The fractional derivative of order $\delta(\delta>0)$ is defined, for a function $f$, analytic in a simply connected region of the complex plane containing the origin, by

$$
D_{z}^{-\delta} f(z)=\frac{1}{\Gamma(\delta)} \int_{0}^{z} \frac{f(\zeta)}{(z-\zeta)^{1-\delta}} d \zeta
$$

where the multiplicity of $(z-\zeta)^{\delta-1}$ is removed by requiring $\log (z-\zeta)$ to be real when $z-\zeta>0$.

Definition 3.2. Under the hypothesis of Definition 3.1, the fractional derivative of $f$ of order $\delta(\delta \geq 0)$ is defined by

$$
D_{z}^{\delta} f(z)= \begin{cases}\frac{1}{\Gamma(1-\delta)} \frac{d}{d z} \int_{0}^{z} \frac{f(\zeta)}{(z-\zeta)^{\delta}} d \zeta, & (0 \leq \delta<1) \\ \frac{d^{n}}{d z^{n}} D_{z}^{\delta-n} f(z), & \left(n \leq \delta<n+1 ; n \in \mathbb{N}_{0}\right)\end{cases}
$$

where $\mathbb{N}_{0}=\mathbb{N} \cup\{0\}$ the multiplicity of $(z-\zeta)^{-\delta}$ is removed as Definition 3.1. 
Definition 3.3. [18] The extended fractional differ integral operator $\Omega_{z}^{\delta}$ : $\mathcal{A}_{p} \rightarrow \mathcal{A}_{p}$ for a function $f$ of the form (1.3) in $\mathcal{U}$ and for a real number $\delta(-\infty<\delta<p+1)$ is defined by

$$
\begin{aligned}
\Omega_{z}^{\delta} f(z) & =z^{p}+\sum_{j=p+1}^{\infty} \frac{\Gamma(j+1) \Gamma(p+1-\delta)}{\Gamma(p+1) \Gamma(j+1-\delta)} a_{j} z^{j} \\
& =z^{p}{ }_{2} F_{1}(1, p+1 ; p+1-\delta ; z) * f(z), \quad(-\infty<\delta<p+1 ; z \in \mathcal{U}) .
\end{aligned}
$$

We note that

$$
\Omega_{z}^{0} f(z)=f(z), \quad \Omega_{z}^{1} f(z)=\frac{z f^{\prime}(z)}{p},
$$

and in general

$$
\Omega_{z}^{\delta} f(z)=\frac{\Gamma(p+1-\delta)}{\Gamma(p+1)} z^{\delta} D_{z}^{\delta} f(z), \quad(-\infty<\delta<p+1 ; z \in \mathcal{U})
$$

where $D_{z}^{\delta} f(z)$ is respectively, the fractional integral of $f(z)$ of order $-\delta$ when $-\infty<\delta<0$ and the fractional derivative of $f(z)$ of order $\delta$ when $0 \leq \delta<$ $p+1$.

Let $\mathcal{C}_{p, q, \delta}^{n, \gamma}(\phi)$ be the class of the functions $f \in \mathcal{A}_{p}$ for which $\Omega_{z}^{\delta} f(z) \in$ $\mathrm{C}_{p, q}^{n, \gamma}(\phi)$. The class $\mathcal{C}_{p, q, \delta}^{n, \gamma}(\phi)$ is the special case of the class $\mathfrak{C}_{p, q, g}^{n, \gamma}(\phi)$, when

$$
g(z)=z^{p}+\sum_{j=p+1}^{\infty} \frac{\Gamma(j+1) \Gamma(p+1-\delta)}{\Gamma(p+1) \Gamma(j+1-\delta)} z^{j} .
$$

Since

$$
\Omega_{z}^{\delta} f(z)=z^{p}+\sum_{j=p+1}^{\infty} \frac{\Gamma(j+1) \Gamma(p+1-\delta)}{\Gamma(p+1) \Gamma(j+1-\delta)} a_{j} z^{j} .
$$

Now applying Theorem 2.8 for the function $(f * g)(z)=z^{p}+a_{p+1} g_{p+1} z^{p+1}+$ $a_{p+2} g_{p+2} z^{p+2}+\cdots$, we get the following theorem.

Theorem 3.1. Let $g(z)=1+g_{1} z+g_{2} z^{2}+\cdots\left(g_{n}>0\right)$. If $f(z)$ given by (1.3) belongs to $\mathrm{e}_{p, q}^{n, \gamma}(\phi)$, then

$$
\left|a_{p+2}-\mu a_{p+1}^{2}\right| \leq \begin{cases}\frac{2 \gamma k_{1}}{g_{p+2}}\left[B_{1}^{2}+B_{2}-\mu \frac{g_{p+2}}{g_{p+1}^{2}} \gamma B_{1}^{2} k_{2}\right], & \text { if } \mu \leq \tau_{1} \\ \frac{2 \gamma B_{1} k_{1}}{g_{p+2}}, & \text { if } \tau_{1} \leq \mu \leq \tau_{2} \\ \frac{-2 \gamma k_{1}}{g_{p+2}}\left[B_{1}^{2}+B_{2}-\mu \frac{g_{p+2}}{g_{p+1}^{2}} \gamma B_{1}^{2} k_{2}\right], & \text { if } \mu \geq \tau_{2} .\end{cases}
$$


where

$$
\tau_{1}=\frac{g_{p+1}^{2}}{g_{p+2} \gamma B_{1} k_{2}}\left[-1+\frac{B_{2}}{B_{1}}+B_{1}\right]
$$

and

$$
\tau_{2}=\frac{g_{p+1}^{2}}{g_{p+2} \gamma B_{1} k_{2}}\left[1+\frac{B_{2}}{B_{1}}+B_{1}\right] .
$$

The result is sharp.

Remark 3.1. Since $\Omega_{z}^{\delta} f(z)=z^{p}+\sum_{j=p+1}^{\infty} \frac{\Gamma(j+1) \Gamma(p+1-\delta)}{\Gamma(p+1) \Gamma(j+1-\delta)} a_{j} z^{j}$,

we have

$$
\begin{gathered}
g_{p+1}=\frac{\Gamma(p+2) \Gamma(p+1-\delta)}{\Gamma(p+1) \Gamma(p+2-\delta)}=\frac{p+1}{p+1-\delta} \\
g_{p+2}=\frac{\Gamma(p+3) \Gamma(p+1-\delta)}{\Gamma(p+1) \Gamma(p+3-\delta)}=\frac{(p+1)(p+2)}{(p+1-\delta)((p+2-\delta)} .
\end{gathered}
$$

For $g_{p+1}, g_{p+2}$ given by (3.5) and (3.6) respectively, Theorem 2.9 reduces to the following Theorem.

Theorem 3.2. Let $\phi(z)=1+B_{1} z+B_{2} z^{2}+\cdots$, where $B_{n}$ 's are real with $B_{1}>0$ and $B_{2} \geq 0$. If $f(z)$ given by(1.3) belongs to $\mathcal{C}_{p, q}^{n, \gamma}(\phi)$, then $\left|a_{p+2}-\mu a_{p+1}^{2}\right| \leq$

$$
\begin{cases}\frac{(p+1-\delta)(p+2-\delta)}{(p+1)(p+2)} 2 \gamma k_{1}\left[B_{1}^{2}+B_{2}-\mu \frac{(p+2)(p+1-\delta)}{(p+1)(p+2-\delta)} \gamma B_{1}^{2} k_{2}\right], & \text { if } \mu \leq \tau_{1}^{*} \\ \frac{(p+1-\delta)(p+2-\delta)}{(p+1)(p+2)} 2 \gamma B_{1} k_{1}, & \text { if } \tau_{1}^{*} \leq \mu \leq \tau_{2}^{*} \\ \frac{-(p+1-\delta)(p+2-\delta)}{(p+1)(p+2)} 2 \gamma k_{1}\left[B_{1}^{2}+B_{2}-\mu \frac{(p+2)(p+1-\delta)}{(p+1)(p+2-\delta)} \gamma B_{1}^{2} k_{2}\right], & \text { if } \mu \geq \tau_{2}^{*}\end{cases}
$$

where

$$
\tau_{1}^{*}=\frac{(p+1)(p+2-\delta)}{(p+2)(p+1-\delta) \gamma B_{1} k_{2}}\left[-1+\frac{B_{2}}{B_{1}}+B_{1}\right]
$$

and

$$
\tau_{2}^{*}=\frac{(p+1)(p+2-\delta)}{(p+2)(p+1-\delta) \gamma B_{1} k_{2}}\left[1+\frac{B_{2}}{B_{1}}+B_{1}\right] .
$$

The result is sharp.

Remark 3.2. It is interesting to note that several well known and new results could be obtained on specializing the function $\phi$ and the parameters involved in Theorem 3.1 and Theorem 3.2 (see $[20,25]$ ). 


\section{Acknowledgement}

The authors thank the referee for valuable comments and suggestions on the earlier version of this paper. Particularly, we thank for comments leading to an improvement in Theorem 2.3.

\section{References}

[1] Ludwig Bieberbach, Über die koeffinzienten derjenigen potenzreihen, welche cine schlichte Abbildung des Einheitskreises wermitteln. S.-B. Preuss. Akad. Wiss., (1916), 940-955.

[2] A. Chonweerayoot, D. K. Thomas, and W. Upakarnitikaset, On the FeketeSzegö theorem for close-to-convex functions, Publ. Inst. Math. (Beograd) (N.S.), 52(66), (1992), 18-26

[3] M. Darus and D. K. Thomas, The Fekete-Szegö theorem for strongly close-toconvex functions, Sci. Math., 3 (2), (2000), 201-212

[4] M. Fekete and G. Szegö, Eine Bemerkung Uber Ungerade Schlichte Funktionen, J. London Math. Soc., S1-8 (2), 85.

[5] A. W. Goodman, Univalent functions. Vol. I, Mariner Publishing Co., Inc., Tampa, FL, 1983.

[6] Ian Graham and Gabriela Kohr, Geometric function theory in one and higher dimensions, Monographs and Textbooks in Pure and Applied Mathematics, Marcel Dekker, Inc., New York, 255, 2003.

[7] M. K. Aouf, H. E. Darwish, and A. A. Attiya, On a class of certain analytic functions of complex order, Indian J. Pure Appl. Math., 32 (10), (2001), 1443-1452.

[8] A. A. Attiya, On a generalization class of bounded starlike functions of complex order, Appl. Math. Comput., 187 (1), (2007), 62-67.

[9] Anatoly A. Kilbas, Hari M. Srivastava, and Juan J. Trujillo, Theory and applications of fractional differential equations, North-Holland Mathematics Studies, Elsevier Science B.V., Amsterdam, 204, 2006

[10] Wolfram Koepf, On the Fekete-Szegő problem for close-to-convex functions, Proc. Amer. Math. Soc., 101 (1), (1987), 89-95

[11] K. Kuroki and S. Owa, Notes on new class for certain analytic functions, RIMS Kokyuroku, 1772 (1), (2011), 21-25.

[12] Wan Cang Ma and David Minda, A unified treatment of some special classes of univalent functions, 1994, 157-169.

[13] William Ma and David Minda, Coefficient inequalities for strongly close-to-convex functions, J. Math. Anal. Appl., 205 (2), (1997), 537-553. 
[14] M. A. Nasr and M. K. Aouf, Starlike function of complex order, J. Natur. Sci. Math., 25 (1), (1985), 1-12.

[15] Shigeyoshi Owa, On the distortion theorems. I, Kyungpook Math. J., 18 (1), (1978), $53-59$.

[16] Shigeyoshi Owa, On applications of the fractional calculus, Math. Japon., 25 (2), (1980), 195-206.

[17] Shigeyoshi Owa and H. M. Srivastava, Univalent and starlike generalized hypergeometric functions, Canad. J. Math., 39 (5), (1987), 1057-1077.

[18] J. Patel and A. K. Mishra, On certain subclasses of multivalent functions associated with an extended fractional differintegral operator, J. Math. Anal. Appl., 332 (1), (2007), 109-122.

[19] V. Ravichandran, Yasar Polatoglu, Metin Bolcal, and Arzu Sen, Certain subclasses of starlike and convex functions of complex order, Hacet. J. Math. Stat., 34, (2005), 9-15.

[20] Fuad S. M. Al Sarari and S. Latha, Fekete-Szego inequalities and $(j, k)$-symmetric functions, Electron. J. Math. Anal. Appl., 3 (2), (2015), 249-256.

[21] Grigore Ştefan Sălăgean, Subclasses of univalent functions, 1983, 362-372.

[22] Stefan G. Samko, Anatoly A. Kilbas, and Oleg I. Marichev, Fractional integrals and derivatives, Gordon and Breach Science Publishers, Yverdon, 1993

[23] A. C. Schaeffer and D. C. Spencer, Coefficient Regions for Schlicht Functions, American Mathematical Society Colloquium Publications, Vol. 35, American Mathematical Society, New York, N. Y., 1950.

[24] T. M. Seoudy and M. K. Aouf, Coefficient estimates of new classes of $q$-starlike and $q$-convex functions of complex order, J. Math. Inequal., 10 (1), (2016), 135-145.

[25] T. N. Shanmugam, C. Ramachandran, and V. Ravichandran, Fekete-Szegö problem for subclasses of starlike functions with respect to symmetric points, Bull. Korean Math. Soc., 43 (3), (2006), 589-598.

[26] Jae Ho Choi, Yong Chan Kim, and Toshiyuki Sugawa, A general approach to the Fekete-Szegö problem, J. Math. Soc. Japan, 59 (3), (2007), 707-727.

[27] Paweł Wiatrowski, The coefficients of a certain family of holomorphic functions, Zeszyty Nauk. Uniw. Lódz. Nauki Mat. Przyrod. Ser. II, 39 Mat., (1971), 75-85.

\section{Selvaraj}

Department of Mathematics,

Presidency College (Autonoumous),

Chennai-600 005, Tamilnadu, India.

E-mail: pamc9439@yahoo.co.in

K. R. Karthikeyan 
Vol. LIV (2016) Fekete-Szegö inequalities ...

Department of Mathematics and Statistics,

Caledonian College of Engineering,

Muscat, Sultanate of Oman.

E-mail: kr_karthikeyan1979@yahoo.com

S. Lakshmi

Department of Mathematics,

R.M.K.Engineering College,

R.S.M.Nagar, Kavaraipettai-601206,

Tamilnadu,India.

E-mail: laxmirmk@gmail.com

Received: 25.03.2016

Accepted: 28.06.2016 